\title{
Daily Imaging Scheduling of An Earth Observation Satellite*
}

\author{
Wei-Cheng Lin \\ Electrical Engineering Department \\ National Taiwan University \\ Taipei 106, Taiwan, R.O.C. \\ byron@ac.ee.ntu.edu.tw \\ Chung-Yang Liu \\ Communication School \\ Ming-Chuan University \\ Taipei, Taiwan, R.O.C. \\ cyliu@mcu.edu.tw
}

\author{
Da-Yin Liao \\ Information Management Department \\ National Chi-Nan University \\ Puli, Nantou 545, Taiwan, R.O.C \\ dyliao@im.ncnu.edu.tw \\ Yung-Yao Lee \\ System Engineering Division \\ National Space Programs Office \\ Hsinchu 300, Taiwan, R.O.C. \\ yylee@nspo.com.tw
}

\begin{abstract}
This paper presents the research and development of a daily imaging scheduling system for a low-orbit, earth observation satellite. This satellite daily imaging scheduling problem belongs to a class of singlemachine scheduling problems with salient features of sequence-dependent setup effects, job-assembly characteristics, and time window constraints. It is NP. hard in computational complexity. We adopt Lagrangian relaxation and linear search techniques to solve this problem. Numerical results demonstrate that this approach is efficient in applications to the real problems. It is very effective to generate a near-optimal, feasible schedule for the imaging operations of the satellite.
\end{abstract}

Keywords: Satellite Scheduling, Lagrangian Relaxation

\section{Introduction}

This paper deals with the research and development of an imaging scheduling system for a low-orbit, earth observation satellite (EOS), ROCSAT-II [10]. Each day, ROCSAT-II will take three to four strips of images in approximately 10 minutes when it traverses the Taiwan island. It takes time and power to maneuver the satellite from its previous position to the desired aspect angle for the new imaging operation. The maneuvering time and power depend on the positions and postures of the two consecutive imaging operations. For a given imaging area, the solution to the position and posture of the spacecraft is not unique. There are preferences for the possible combination of positions and postures. They are represented in a form of "window of opportunity," specified by a suitability function [9]. This scheduling problem becomes to generate an imaging schedule, while considering the priority of imaging requests, the coverage of clouds, the resource availability of the spacecraft like power, space for data storage, and the physical limitation of camera turning angles. An imaging request may involve more than one imaging operation to fulfill the request.

*This work is supported in part by National Space Project Office, R.O.C., under grant of NSC91-NSPO (A)-PC-FA12-01.

* 0-7803-7952-7/03/\$17.00 $\odot 2003$ IEEE.
This satellite imaging scheduling problem belongs to a class of single-machine scheduling problems featured by sequence-dependent setup effects, jobassembly characteristics, and time window constraints, which is NP-hard in computational complexity [6]. The study of scheduling problems with sequence dependent setup has attracted quite an amount of attention for years [6]. For problems of such a high complexity, dynamic programming and exhaustive search techniques are either too time-consuming or impractical to solve for the optimal solution. Rule-based or heuristics approaches can reduce the computation time drastically. However, the resultant optimality is not guaranteed.

Agnese et al [1] deal with the daily photographscheduling problem of an earth observation satellite system like SPOT [12]. This problem can be viewed as an instance of the valued constraint satisfaction problem framework, which can solved by the exact methods like the depth-first branch-and-bound or pseudo dynamic search, or by approximate methods, like greedy search or Tabu search. Vasquez and Hao [8] formulate the daily photograph-scheduling problem of SPOT as a generalized version of the knapsack model and solve by a dedicated Tabu search algorithm. Wolfe and Sorensen [9] define and use the "window-constrained packing" problem to model the NASA's earth observation system domain scheduling problem. They propose three algorithms: a dispatch algorithm, a look-ahead algorithm, and a genetic algorithm, which can only be applied to a limited and static part of the EOS problem.

LANDSAT-7 [11] is the newest member of the LANDSAT family of remote sensing satellite. Porter and Gasch [7] propose an image scheduler for LANDSAT-7, which uses a multi-pass scheduling algorithm. Their scheduling algorithm employs rules based on optimistic resource allocation and look-behind preemption to adjust past decisions based on current knowledge. This algorithm is a linear finite deterministic model. The image scheduler is not an optimal scheduler because it fails to execute full backtracking to find the most costeffective path solution. 
Instead of pursuit for the optimal solution, in the paper, a mathematic programming approach is adopted to achieve a near-optimal solution with allowable computation time, which is very effective for scheduling problems in large-scaled or time-critical practices. The daily imaging scheduling problem is first formulated as an integer-programming problem. We then use the Lagrangian relaxation technique to decompose the daily imaging scheduling problem into individual task-level scheduling subproblems. Given a set of Lagrangian multipliers, each subproblem is solved by a linear search method. A dual function is formed to optimize the Lagrangian multipliers by a subgradient method [3]. A heuristic algorithm based on the dual solution is then developed to find a near-optimal and feasible solution.

The remainder of this paper is organized as follows. Section 11 describes the mathematical modeling of the satellite daily imaging scheduling problem. Solution methodology and development of a feasibility adjustment heuristic are described in Section III. Section IV conducts the numerical experiments and demonstrates its ability in the applications to ROCSAT-Il image acquisition scheduling. Finally, in Section V concluding remarks are made with some future research directions.

\section{Satellite Scheduling Problem Formulation}

Define a task to be a basic operation of image acquisition over an area of the earth. Since an imaging request may need more than one tasks, let a job be the collection of all the tasks to fulfill the request. Some assumptions are made as follows.

1. A task can belong to a job only.

2. A task can only be processed at most once during the scheduling time horizon.

3. Only a task is being processed or being setup at a time.

4. All the imaging requests are released and given at the beginning of the scheduling time horizon.

5. There are $N$ distinct areas with cloud coverage above them during the scheduling time horizon.

Let us define some notations for modeling the satellite daily imaging scheduling problem.

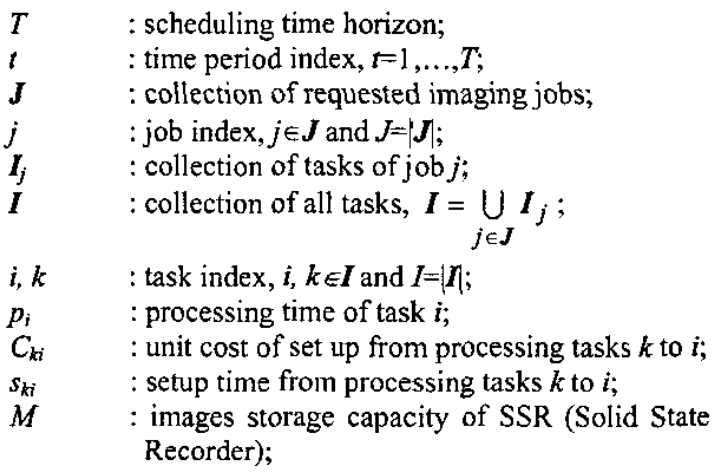

D : available power before the imaging operation begins;

$m_{i} \quad$ : imaging mode of task $i, m_{i} \in\{$ Panchromatic (PAN), Multi-Spectral (MS), PAN+MS \};

$q_{i} \quad:$ image size of task $i$;

$u_{i} \quad$ : power required for processing task $i$;

$v_{k i} \quad$ : power required for setup from processing task $k$ to $i$;

$\left\lfloor w_{i}^{b}, w_{i}^{e}\right\rfloor:$ opportunity window of task $i, w_{i}^{b}$ and $w_{i}^{e}$ are the time frame available for processing task $i$;

$\Gamma_{i}(t) \quad$ : suitability of task $i$ at time $t$

$A_{j} \quad$ : penalty of incompleteness of job $j, A_{j} \geq 0$;

$B_{i} \quad:$ suitability benefit of task $i, B_{i} \geq 0$;

$\left\lfloor w_{n}^{b}, w_{n}^{e}\right\rfloor:$ the $n$th cloud coverage area, $n=1, \ldots, N$;

$b_{i} \quad:$ the time when task $i$ starts its processing;

$\alpha_{i t} \quad:$ a step function indicating that task $i$ is processed, where $\alpha_{i t}=\left\{\begin{array}{l}1, \forall t \geq b_{i} \\ 0, \forall t<b_{i}\end{array} ;\right.$

$\beta_{j} \quad:$ a binary variable indicating that job $j$ is complete, where

$$
\beta_{j}=\left\{\begin{array}{l}
0, \text { if } \alpha_{i}\left(T-p_{i}+1\right)=1, \forall i \in \mathbf{I}_{j} ; \\
1, \text { otherwise }
\end{array} ;\right.
$$

$\gamma_{k i t}$

: decision variable for setup from processing tasks $k$ to $i$, where $\gamma_{k i t}=\left\{\begin{array}{l}1, \forall t \geq b_{i}-s_{k i} \\ 0, \text { otherwise }\end{array} ;\right.$

Initial Setup Constraint: Assume that the initial state of the satellite is setup to a dummy task, 0 , where, $s_{0 i}=0$, $C_{0 i}=0$, and $v_{0 i}=0, \forall i \in I$. As there is one and only one task that can be setup from task 0 , we have

$$
\sum_{i=1}^{I} \gamma_{0 i T}=1
$$

Setup Constraints: An imaging operation cannot commence its processing before completing its setup. We have

$$
\alpha_{i t}=\sum_{\substack{k=0 \\ k \neq i}}^{I} \gamma_{k i\left(t-s_{k i}\right)}, \quad \forall i, t
$$

Machine Capacity Constraints: Since there is only one camera equipped with the satellite, at any time, there is at most one task being processed or setup on the satellite, that is,

$$
\sum_{i=1}^{l}\left[\alpha_{i t}-\alpha_{i\left(t-p_{i}\right)}\right]+\sum_{\substack { i=1 \\
\begin{subarray}{c}{k=0 \\
k \neq i{ i = 1 \\
\begin{subarray} { c } { k = 0 \\
k \neq i } }\end{subarray}}^{l}\left[\gamma_{k i t}-\gamma_{k i\left(t-s_{k i}\right)}\right] \leq 1, \forall t .
$$

Storage Capacity Constraint: The images acquired are first stored on board until they can be downloaded towards a ground station. As the total available memory on board is limited, this may impose constraints on the selection of images as well as their scheduling. The total size of images taken should be less than the available image storage capacity before imaging operations take 
place. Note that the coefficient $q_{i}$ is related to the adopted imaging mode.

$$
\sum_{i=1 t=1}^{I} \sum_{t=1}^{T} q_{i}\left(\alpha_{i t}-\alpha_{i\left(t-p_{i}\right)}\right) \leq M
$$

Power Consumption Constraint: The total power consumption for imaging and setup operations should be less than the available power, $D$.

$$
\left.\sum_{i=1 t=1}^{I} \sum_{i}^{T} u_{i}\left[\alpha_{i t}-\alpha_{i\left(t-p_{i}\right)}\right]+\sum_{\substack{i=1 \\ k \neq 0 \\ k \neq i}}^{I} \sum_{\substack{k=1 \\ k}}^{T} \gamma_{k i}\left[\gamma_{k i t}-\gamma_{k i\left(t-s_{k i}\right.}\right)\right] \leq D
$$

Window of Cloud Coverages: The mission of ROCSATII is to acquire substantially cloud-free images. We accomplish this by employing cloud coverage prediction data sets from the weather forecast data of Center Weather Bureau (CWB). Any task that intends to take images of an area with cloud coverage is assumed invalid.

$$
b_{i} \notin\left[w_{n}^{b}-p_{i}, w_{n}^{e}\right\rfloor \quad \forall i, n=1, \ldots, N
$$

Binary Constraints: As the variables $\alpha, \beta$, and $\gamma$ are all of either 0's or l's, the following binary constraints should be satisfied.

$$
\begin{array}{ll}
\alpha_{i t} \in\{0,1\}, & \forall i \in I, t . \\
\beta_{j} \in\{0,1\}, & \forall j \in J . \\
\gamma_{k i t} \in\{0,1\}, & \forall k, i \in I, t .
\end{array}
$$

Our objective of the imaging scheduling problem has three folds: the first one is to minimize the weighted number of incomplete jobs, as defined as follows:

$$
\sum_{j=1}^{J} A_{j} \beta_{j}
$$

Another objective considers the suitability benefits of imaging within the window of opportunity, $\left[w_{i}^{b}, w_{i}^{e}\right] \forall i$. For a task, different-level of performance can be achieved at the different location within its window of opportunity, due to satellite dynamics and its aspect angle to the target of interest. A suitability function, $\Gamma \mathrm{i}(\mathrm{t})$, is thus defined for the suitability of executing task $\mathrm{i}$ over the time horizon. An example of the suitability function is shown in Fig. 1. The operation period represents for the imaging period.

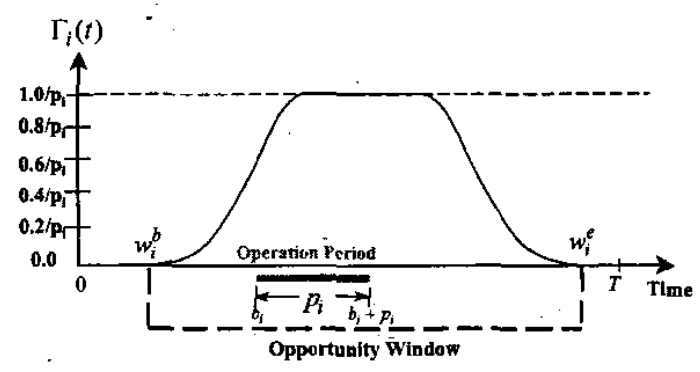

Fig. 1. Suitability Function $\Gamma_{i}(t)$
For task i, its resultant suitability is $\sum_{t=1}^{T} B_{i} \Gamma_{i}(t)\left[\alpha_{i t}-\alpha_{i\left(t-p_{i}\right)}\right)$. Our objective is to maximize the total weighted suitabilities of all the tasks, i.e.,

$$
\sum_{i=1 t=1}^{I} \sum_{t=1}^{T} B_{i} \Gamma_{i}(t)\left[\alpha_{i t}-\alpha_{i\left(t-p_{i}\right)}\right)
$$

The other objective of the scheduling problem is to minimize the total setup costs incurred,

$$
\sum_{\substack{i=1 \\ k \neq 1 \\ k \neq i}}^{I} \sum_{\substack{t=1 \\ k}}^{T} C_{k i}\left[\gamma_{k i t}-\gamma_{k i\left(t-s_{k i}\right)}\right]
$$

The satellite imaging scheduling problem is then to schedule imaging operation that minimizes the weighted number of incomplete jobs and the total operational setup costs, and maximizes the total suitability benefits of all the tasks while satisfying all the constraints. Mathematically, it is formulated as

$$
\begin{aligned}
\min _{\gamma} & \sum_{j=1}^{J} A_{j} \beta_{j}-\sum_{i=1}^{I} \sum_{t=1}^{T} B_{i} \Gamma_{i}(t)\left[\alpha_{i t}-\alpha_{i\left(t-p_{i}\right)}\right] \\
+ & \sum_{\substack{i=1 \\
k}}^{I} \sum_{\substack{k=0 \\
k \neq i}}^{I} \sum_{t=1}^{T} C_{k i}\left[\gamma_{k i t}-\gamma_{k i\left(t-s_{k i}\right)}\right]
\end{aligned}
$$

subject to constraints (1) to (7).

In (P), the first term is for the weighted number of incomplete jobs, the second for preferences for placements within the window of opportunity, and the final for the costs incurred by setup operations.

\section{Solution Methodology}

The scheduling problem (P) formulated in Section II is an integer linear programming problem of NP-hard computational complexity [6]. As there are couplings of tasks in $\sum_{j=1}^{J} A_{j} \beta_{j}$, which make the difficulties in solving the problem, thus we develop an approximation to this term that yields a lower bound to (P).

Let $\bar{A}_{i}=A_{j}, \forall i \in I_{j}$, and let $\bar{I}_{i}=\left|\boldsymbol{I}_{j}\right|, \forall i \in \boldsymbol{I}_{j}$. We then have the following two lemmas.

Lemma 1: $\quad \sum_{j=1}^{J} A_{j}=\sum_{i=1}^{I}\left(\frac{\bar{A}_{i}}{\bar{I}_{i}}\right)$.

Proof: As a task can belong to a job only, we have

$$
\begin{aligned}
& \sum_{i=1}^{I}\left(\frac{\bar{A}_{i}}{\bar{I}_{i}}\right)=\sum_{j=1}^{J} \sum_{i \in \mathbf{I}_{j}}\left(\frac{\bar{A}_{i}}{\bar{I}_{i}}\right)=\sum_{j=1}^{J} \sum_{i \in \mathbf{I}_{j}}\left(\frac{A_{j}}{\left|\mathbf{I}_{j}\right|}\right)=\sum_{j=1}^{J} \frac{A_{j}}{\left|\mathbf{I}_{j}\right|} \sum_{i \in \mathbf{I}_{j}}(1) \\
& =\sum_{j=1}^{J} A_{j}
\end{aligned}
$$


Lemma 2: $\quad \beta_{j} \geq 1-\frac{1}{\left|\mathbf{I}_{j}\right|} \sum_{i \in \mathbf{I}_{j}} \alpha_{i\left(T-p_{i}+1\right)}, \forall j$.

Proof: From the definition of $\beta_{j}$,

$$
\beta_{j}=1-\left\lfloor\left.\frac{1}{\mid \mathbf{I}}\right|_{j} \mid i \in \mathbf{I}_{j} \alpha_{i\left(T-p_{i}+1\right)}\right\rfloor
$$

Since $x-1<\lfloor x\rfloor \leq x, \forall x \in \mathfrak{R}$, we have

$$
\frac{1}{\left|\mathbf{I}_{j}\right|} \sum_{i \in \mathbf{I}_{j}} \alpha_{i\left(T-p_{i}+1\right)}-1<\left\lfloor\frac{1}{\left|\mathbf{I}_{j}\right|} \sum_{i \in \mathbf{I}_{j}} \alpha_{i\left(T-p_{i}+1\right)}\right\rfloor \leq \frac{1}{\left|\mathbf{I}_{j}\right|} \sum_{i \in \mathbf{I}_{j}} \alpha_{i\left(T-p_{i}+1\right)} .
$$

Therefore,

$$
\beta_{j} \geq 1-\frac{1}{\left|\mathbf{I}_{j}\right|} \sum_{i \in \mathbf{I}_{j}} \alpha_{i\left(T-p_{i}+1\right)}, \forall j
$$

Theorem 1: $\sum_{j=1}^{J} A_{j} \beta_{j} \geq \sum_{i=1}^{L}\left(\frac{\vec{A}_{i}}{\bar{I}_{i}}\right)-\sum_{i=1}^{I}\left(\frac{\bar{A}_{i}}{\bar{I}_{i}}\right) \alpha_{i\left(T-p_{i}+1\right)}$

Proof: From lemmas 1 and 2,

$$
\begin{aligned}
\sum_{j=1}^{J} A_{j} \beta_{j} & \geq \sum_{j=1}^{J} A_{j}\left(1-\frac{1}{\left|\boldsymbol{I}_{j}\right|} \sum_{i \in I_{j}} \alpha_{i\left(T-p_{i}+1\right)}\right) \\
= & \sum_{i=1}^{I}\left(\frac{\bar{A}_{i}}{\bar{I}_{i}}\right)-\sum_{j=1}^{J} A_{j} \sum_{i \in I_{j}}\left(\frac{1}{\left|\boldsymbol{I}_{j}\right|}\right) \alpha_{i\left(T-p_{i}+1\right)} \\
= & \sum_{i=1}^{I}\left(\frac{\bar{A}_{i}}{\bar{I}_{i}}\right)-\sum_{i=1}^{I}\left(\frac{\bar{A}_{i}}{\bar{I}_{i}}\right) \alpha_{i\left(T-p_{i}+1\right)} \\
& =\sum_{i=1}^{I}\left(\frac{\bar{A}_{i}}{\bar{I}_{i}}\right)\left(1-\alpha_{i\left(T-p_{i}+1\right)}\right)
\end{aligned}
$$

From theorem 1, define a new mathematical program $\left(\boldsymbol{P}^{\prime}\right)$ as

$\left(\mathbf{P}^{\prime}\right)$

$$
\begin{aligned}
& \min _{\gamma} \sum_{i=1}^{I}\left(\frac{\bar{A}_{i}}{\bar{I}_{i}}\right)\left[1-\alpha_{i\left(T-p_{i}+1\right)}\right]-\sum_{i=t t=1}^{I} \sum_{i}^{T} B_{i} \Gamma_{i}(t)\left[\alpha_{i t}-\alpha_{i\left(t-p_{i}\right)}\right] \\
& +\sum_{\substack{i=1 \\
k \neq=}}^{I} \sum_{\substack{k \\
k \neq i}}^{T} \sum_{t=1} C_{k i}\left[\gamma_{k i t}-\gamma_{k i\left(t-s_{k j}\right)}\right]
\end{aligned}
$$

subject to constraints (1) to (7).

Note that solutions to $\left(\boldsymbol{P}^{\prime}\right)$ provide a lower bound to those of $(\mathrm{P})$.

We then adopt the Lagrangian relaxation approach along with the linear search technique to solve problem $\left(\boldsymbol{P}^{\prime}\right)$. Solution development is then detailed as follows.

\subsection{Decomposition by Lagrangian Relaxation}

In problem $\left(\boldsymbol{P}^{\prime}\right)$, we first apply the Lagrangran relaxation technique to initial setup constraint (1), machine capacity constraints (3), storage capacity constraint (4), and power consumption constraint (5) with Lagrangian multipliers $\xi,\left\{\lambda_{t}, \forall t\right\}, \pi$, and $\eta$, respectively, where $\xi \in R$ and $\lambda_{t} \geq 0, \forall t, \pi \geq 0, \eta \geq 0$. We form a Lagrangian function $\Phi(\xi, \lambda, \pi, \eta)$ for problem $\left(\boldsymbol{P}^{\prime}\right)$ and decompose into task-level subproblem $\Phi_{i}(\xi, \lambda, \pi, \eta), \forall i \in \boldsymbol{I}$, as follows:

$$
\Phi(\xi, \lambda, \pi, \eta)=\sum_{i=1}^{I} \Phi_{i}(\xi, \lambda, \pi, \eta)+\sum_{i=1}^{I}\left(\frac{\bar{A}_{i}}{\bar{I}_{i}}\right)-\xi-\sum_{t=1}^{T} \lambda_{t}-\pi M-\eta D .
$$

The dual problem (D) to $\left(\boldsymbol{P}^{\prime}\right)$ after Lagrangian relaxation can be defined in the following

$$
\begin{aligned}
& \text { (D) } \max _{\xi, \lambda, \lambda, \eta}\left\{\min _{y} \Phi(\xi, \lambda, \pi, \eta)\right\} \\
& \equiv \max _{\xi, \lambda, \pi, \eta}\left\{\sum_{i=1}^{I} \min \Phi_{i}(\xi, \lambda, \pi, \eta)+\sum_{i=1}^{I}\left(\frac{\bar{A}_{i}}{\bar{I}_{i}}\right)-\xi-\sum_{i=1}^{T} \lambda_{i}-\pi M-\eta D\right\}
\end{aligned}
$$

subject to

$$
\begin{aligned}
& \xi \in R, \\
& \lambda_{t} \geq 0, \quad \forall t, \text { and } \pi \geq 0, \quad \eta \geq 0 .
\end{aligned}
$$

\subsection{Solution Methodology for Subproblems}

The problem $\left(\boldsymbol{P}^{\prime}\right)$ has been decomposed into I subproblems. Each subproblem $\left(\boldsymbol{P}_{i}^{\prime}\right)$ then becomes to determine $\left\{y_{k i t}\right\}, \forall k, t$, in order to minimize the objective function $\Phi_{i}(\xi, \lambda, \pi, \eta)$ of $\left(\boldsymbol{P}_{i}^{\prime}\right)$, while satisfying constraints (2), (6), and (7). As for a given task, say $i$, there exists only one solution to $\gamma_{k i t}=1$, for some $k$ and $t$. Therefore, a linear search method is adopted to find the solution, which yields $\mathrm{O}(I T)$ of computational time.

\subsection{Dual Solutions}

The dual value of $\Phi(\xi, \lambda, \pi, \eta)$ can be calculated by solving all the subproblems for a given set of Lagrangian multipliers, $\{\xi, \lambda, \pi, \eta\}$. Due to the integral requirements in subproblems, the dual function is polyhedral concave made up of many facets and is non-differentiable. Lagrangian multipliers $\xi, \lambda, \pi$ and $\eta$ are iteratively updated by the subgradient method (SG) [3] to solve the dual problem (D). SG is commonly adopted to solve the scheduling problem of realistic sizes [5]. Compared with the other multiplier adjustment methods, SG requires the minimization of all the subproblems to obtain a search direction, which provides an approach for speeding up computation [5]. 


\subsection{Feasibility Adjustment Method}

Theoretically, even when the optimal solution to the dual problem (D) is obtained, it may still result in an infeasible schedule, i.e., some of the constraints (1), (3), (4) and (5) cannot be satisfied by the dual optimal solution. This is because of the integer decision variables involved. However, the dual cost, the minimal cost of a relaxed problem $\left(\boldsymbol{P}^{\prime}\right)$, does provide a lower bound to the optimal cost of $\left(\boldsymbol{P}^{\prime}\right)$. To complete our solution methodology, an iterative heuristic algorithm is further developed to adjust the dual solution to a near-optimal, feasible schedule by taking advantage of the marginal cost interpretation of Lagrangian multipliers.

The heuristic algorithm includes five major steps summarized as follows. The algorithm first resolves the violations on relaxed constraints based on the dual solution. After solving constraint violation and removing incomplete jobs, the processing sequence has been changed. New setup relationships are built. With the help of Lagrangian multipliers, only a complete job with the minimum cost is inserted into the final schedule list and those incomplete jobs are removed. This step continues until no more unscheduled job can be inserted. Finally, for better imaging quality of each scheduled imaging task, fine tune of the schedule is performed to achieve its best feasible solution within its opportunity window.

This heuristic guarantees the feasibility of the final solution but not the optimality. Once a feasible schedule is obtained, the corresponding cost of the objective function is an upper bound on the optimal cost, while the dual cost serves as a lower bound. The difference between the optimal cost and the lower bound is known as the duality gap, which provides a measure of the optimality of the feasible solution; the smaller the gap, the closer the feasible schedule to the optimal.

\section{Numerical Experiments}

Numerical experimentation is conducted in this section to assess the feasibility, optimality, and features of the proposed satellite daily imaging scheduling algorithm. Features of job assembly, setup operation effects, cloud coverage areas, and opportunity windows are considered in the test cases. The algorithm is first applied to a toy example of only two jobs of three tasks with time horizon of six periods, whose optimal schedule can be obtained by using the exhaustive search method. Second, a projected daily imaging scenario of ROCSATII to demonstrate its applicability to the realistic problem. The scheduling algorithm is implemented in $C$ language. All the experiments are conducted in an AMD Athlone$1600 \mathrm{TM}$ PC with memory size of $256 \mathrm{MB}$. All the Lagrangian multipliers are initialized as zero.

\subsection{Test of A Toy Example}

Consider a simple test case where there are only two jobs. Job 1 has only one imaging task while job 2 has two. All the test data of these two jobs are shown in Table 1. The suitability function for each task is illustrated, respectively, in Fig. 2, where the cloud coverage conditions (in dashed slots) are shown as well.

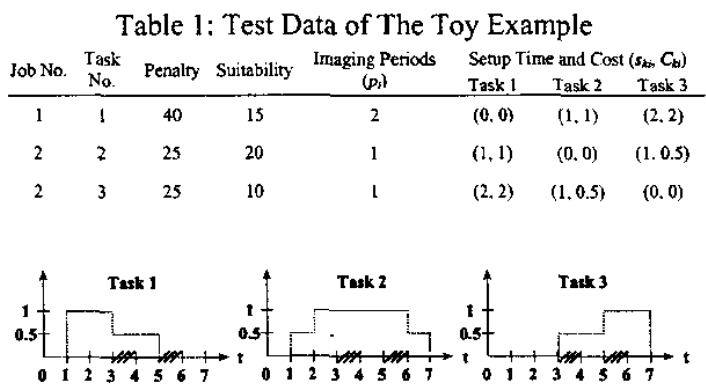

Fig. 2. Suitability Function and Cloud Coverage of The Toy Example

It takes less than one CPU second for the algorithm to generate a daily imaging schedule as shown in the Gantt chart of Fig. 3, which is also the optimal schedule obtained by the exhaustive search on the entire solution space. The resultant feasible cost is -58.5 while the dual cost is -59 . The duality gap, defined as (feasible cost dual cost)/dual cost $\times 100 \%$, is $0.847 \%$. The non-zero duality gap is caused by the same penalty function for task 2 and task 3 , where Lagrangian relaxation is weak to resolve such a tie. In this case, even though the dual solution converges to the dual optimal, the duality gap still exists.

\begin{tabular}{|c|c|c|c|c|c|c|}
\hline Task 2 & \multicolumn{6}{|c|}{ 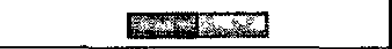 } \\
\hline Task 3 & \multicolumn{6}{|c|}{ Findw } \\
\hline Time Slots & 1 & 2 & 3 & 4 & 5 & 6 \\
\hline
\end{tabular}

Fig. 3. Optimal Solution for The Toy Example

\subsection{Daily Imaging Scheduling of ROCSAT-II}

A daily imaging scheduling problem of ROCSAT-II is designed as depicted in Fig. 4. There are five imaging jobs, Jobs 1 5, which are composed of nine tasks. These five imaging jobs simulate the conditions of widespread tasks (Job 1), large-area tasks (Job 2), separate-but-in-asame-strip tasks (Job 3), cross-stripped tasks (Job 4), and consecutive tasks (Job 5), respectively. The time window of each task is determined by the geographical limitation of the task. The setup time between any two tasks is assumed to be proportional to their geographical distance, and the setup cost is assumed to be twice the value of the corresponding setup time. The scheduling time horizon is of 100 time periods. Two cloud coverage areas are assumed with totally 4 time periods of invalid imaging operations. Three test cases of light, heavy and overloaded loadings are designed with their overall imaging loadings of 67,80 and 93 , respectively. 


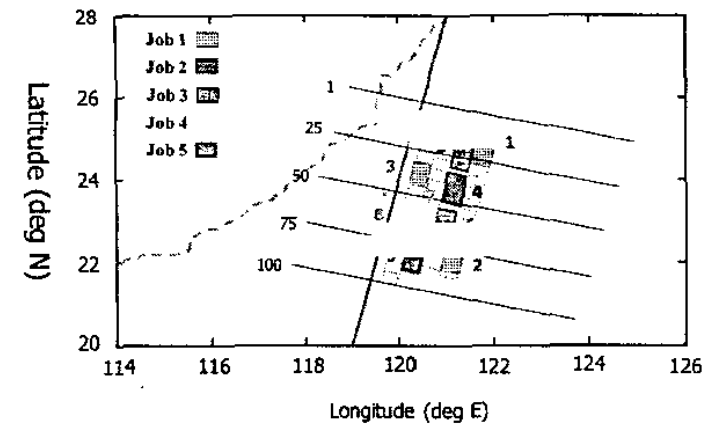

Fig. 4. A Projected Case of 5 Jobs and 9 Tasks in 100 Time Periods

Numerical results of these three test cases are summarized in Table 2 . The duality gaps in both cases of light and heavy loadings are less than $2 \%$, which can be considered near optimal. However, the duality gap in the overloaded case becomes unaffordable large. In fact, solutions to the overloaded case are infeasible. That is, there exists no feasible schedule where all the tasks can be scheduled within the time horizon. In addition to the duality gaps, we adopt the performance measure of PSTC (percentage of setup time consumption) [4] to study the significance of setup time effects on the overall scheduling problem. PSTC is defined as the percentage of total time consumptions for setup against the total available machine time of the time horizon. From Table 2 , the PSTCs are less than $10 \%$ for both the light and heavy cases.

Table 2: Experiment Result of 5 Jobs, 9 Tasks in 100

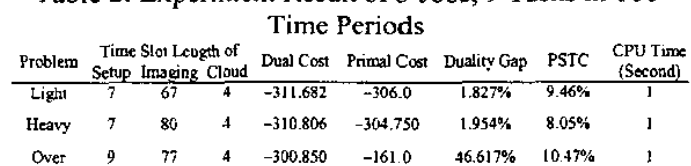

\section{Concluding Remarks}

In this paper, we present the development of a daily imaging scheduling of an earth observation satellite, ROCSAT-1I. The satellite daily imaging scheduling problem belongs to single-machine scheduling problem with sequence-dependent setup effects, job-assembly characteristics, and time window constraints. We utilize Lagrangian relaxation to decompose the problem into independent subproblems, each of which can be easily solved by the efficient linear search method. A heuristic algorithm is developed to find a near-optimal, feasible schedule for daily imaging operations of the satellite. Numerical results demonstrate that our approach is quite effective to the application to the real problem.

Future research may extend the algorithm to include realistic issues such as seasonal refreshment and coordination between multiple satellites. On the other hand, the developed algorithm deals with the scheduling problem assuming no machine failure effects and absolute cloud filters. Extensions to this research involve the development of solution methodologies to handle these stochastic issues.

\section{Acknowledgement}

The authors would thank Dr. Shi-Chung Chang, Dr. An-Ming Wu, and colleagues in NSPO for their valuable suggestion to this project.

\section{References}

[1] J.C. Agnèse, N.Bataille, D. Blumstein, E. Bensana, and G. Verfaillie, "Exact and Approximate Methods for the Daily Management of A Remote Sensing Satellite," Artificial Intelligence and Knowledge Based Systems in Space, ESTEC, 1995.

[2] Virginie Gabrel and Daniel Vanderpooten, "Enumeration and Interactive Selection of Efficient Paths in A Multiple Criteria Graph for Scheduling An Earth Observing Satellite", European Journal of Operation Research, Vol. 139, pp. 533-542, 2002.

[3] M. Held, P. Wolfe, and H. Crowder, "Vaildation and Subgradient Optimization," Math. Programming, Vol. 6, pp. 62-88, 1974.

[4] Chung-Yang Liu; Shi-Chung Chang, "Scheduling flexible flow shops with sequence-dependent setup effects," IEEE Transactions on Robotics and Automation, Vol. 16, Issue 4, pp. 408-419, 2000.

[5] P. B. Luh and D. J. Hoitomt, "Scheduling of Manufacturing Systems Using the Lagrangian Relaxation Technique," IEEE Transactions on Automatic Control, Vol. 38, No. 7, pp. 1066-1079, 1993.

[6] M. Pinedo, "Scheduling Theory, Algorithms, and Systems", Prentice Hall 1995.

[7] W. Potter and J. Gasch, "A Photo Album of Earth: Scheduling LANSDAT 7 Mission Daily Activities," Paper ID: 2b0I0, NASA, 2001.

[8] M. Vasquez and J.-K. Hao, "A Logic-Constrained Knapsack Formulation and A Tabu Algorithm for the Daily Photograph Scheduling of An Earth Observation Satellite," Computational Optimization and Applications 20, No. 2, pp. 137-157, 2001.

[9] W. J. Wolfe and S. E. Sorensen, "Three Scheduling Algorithms Applied to the Earth Observing Systems Domain," Management Science 2000 INFORMS, Vol. 46, No. 1, pp. 148-168, January 2000.

[10]NSPO: http://www.nspo.gov.tw/.

[11]LANDSAT 7: http://landsat.gsfc.nasa.gov/.

[12] Spot lmage Spot 5: http://www.spotimage.fr/home/ system/future/spot $5 /$ welcome.htm. 